\title{
O Projeto Terapêutico Singular e as práticas de saúde mental nos Núcleos de Apoio à Saúde da Família (NASF) em Guarulhos (SP), Brasil
}

\author{
The Singular Therapeutic Project and mental health practices \\ at Family Health Support Centers in Guarulhos \\ in the state of São Paulo, Brazil
}

Alice Ayako Hori ${ }^{1}$

Andréia de Fátima Nascimento ${ }^{1}$

${ }^{1}$ Departamento de Medicina Social, Diretoria da Faculdade, Faculdade de Ciências Médicas da Santa Casa de São Paulo. R. Dr. Cesário Mota Júnio 61, Vila Buarque. 01.221020 São Paulo SP Brasil. aliceayako@ig.com.br

\begin{abstract}
Various studies describe the Singular Therapeutic Project (STP) as a powerful instrument of care for the users of specialized mental health services. It is also put forward as a tool to organize and support the activities of the Family Health Support Centers (FHSC), based on the concepts of co-responsibility and comprehensive care. The article seeks to analyze the development of STP by mental health teams of FHSC and its articulations with services of Primary Health Care (PHC), psychosocial services and other sectors of society. It involved qualitative research performed in Guarulhos in the state of São Paulo in 2012 by systematic observation and conducting semi-structured interviews with mental health professionals from support centers. The content analysis method was employed for interpretation of the findings. The STP in FHSC faces obstacles related to lack of definition of work object (attention or management?), precarious working conditions and overlapping functions. It is necessary to analyze the technological feasibility of the STP based on the peculiarities of $\mathrm{PHC}$, avoiding straightforward transposition of technological tools among the different services that comprise the psychosocial care network.
\end{abstract}

Key words Primary health care, Mental health, Qualitative research, Health service evaluation
Resumo Diversos estudos descrevem o Projeto Terapêutico Singular (PTS) como um instrumento potente de cuidado aos usuários de serviços especializados de saúde mental. Ele também é proposto como ferramenta de organização e sustentação das atividades do Núcleo de Apoio à Saúde da Família (NASF), baseadas nos conceitos de corresponsabilização e gestão integrada do cuidado. O objetivo deste artigo é analisar a elaboração de PTS pelas equipes de saúde mental dos NASF e suas articulações com serviços da Atenção Primária à Saúde (APS), da Atenção Psicossocial e com outros setores da sociedade. A pesquisa, do tipo qualitativa, foi conduzida no municipio de Guarulhos (SP), em 2012, mediante realização de entrevistas semiestruturadas, com profissionais de saúde mental de núcleos de apoio e observação sistemática. $O$ método empregado na interpretação foi análise de conteúdo. O PTS nos NASF esbarra em obstáculos relacionados à indefinição de objeto de trabalho (atenção ou gestão?), à precariedade das condições de trabalho e sobreposição de funçães em torno desse dispositivo. É necessário analisar a viabilidade tecnológica do PTS a partir das peculiaridades da APS, evitando a mera transposição de ferramentas tecnológicas entre diferentes serviços da rede de atenção psicossocial.

Palavras-chave Atenção primária à saúde, Saúde mental, Pesquisa qualitativa, Avaliação de serviços de saúde 


\section{Introdução}

Os profissionais de saúde atuantes na Atenção Primária à Saúde (APS) precisam muitas vezes lidar com situações de adoecimento em um cotidiano de duras e violentas condições sociais ${ }^{1}$. Em consonância com os princípios do Sistema Único de Saúde (SUS), eles têm sido convocados a estruturar trabalhos e a transformar modos de fazer visando o atendimento integral, priorizando as atividades preventivas e mantendo os serviços assistenciais. Como parte dessa abordagem integral, a atenção à saúde mental passou a pôr em prática as transformações propostas pelos ideais da Reforma Psiquiátrica.

A ampliação de ações de saúde mental na APS, no Brasil, foi antecedida por diretrizes internacionais, como a publicação do documento $L a$ introducción de un componente de salud mental en la atención primaria, pela Organização Mundial de Saúde (OMS), em que é enfatizado o papel da saúde mental na efetividade dos serviços de $\mathrm{APS}^{2}$, e reforçada pela publicação de dez recomendações para o enfrentamento dos problemas de saúde mental ${ }^{3}$. Esses documentos destacam a importância do cuidado em saúde mental devido à alta prevalência de transtornos mentais, neurológicos e por uso abusivo de drogas em todas as regiões do mundo e por serem fatores que contribuem para a morbidade e mortalidade prematuras.

O fortalecimento da APS no país tem sido realizado prioritariamente através da Estratégia Saúde da Família (ESF), com o estabelecimento de objetivos, metas e indicadores específicos. Visando apoiar, qualificar e complementar o trabalho das equipes de Saúde da Família - SF, em janeiro de 2008, o Ministério da Saúde - MS promulgou a Portaria 154/GM, criando os Núcleos de Apoio à Saúde da Família (NASF) ${ }^{4}$. Os núcleos de apoio seguem as diretrizes estabelecidas para a APS: ação interdisciplinar e intersetorial, educação em saúde, integralidade, territorialidade, equidade, participação social, humanização e promoção da saúde. Classificados em duas modalidades, de acordo com a abrangência da ESF, critérios populacionais e composição dos profissionais dos núcleos, a atuação dos NASF foi dividida em nove áreas estratégicas. Cabe ao gestor definir o elenco dos profissionais, de acordo com estudos epidemiológicos locais.

A proposta de trabalho do NASF deve ser direcionada à corresponsabilização e à gestão integrada do cuidado (por meio de atendimento compartilhado e interdisciplinar), à troca de saberes e à pactuação de projetos terapêuticos que incluam os usuários dos serviços e a comunidade como participantes dessa construção. Com relação à saúde mental, a portaria recomenda que "tendo em vista a magnitude epidemiológica dos transtornos mentais", cada NASF deva contar com pelo menos um profissional da área de saúde mental (psicólogo, psiquiatra ou terapeuta ocupacional) ${ }^{4}$.

As diretrizes dos NASF descrevem a pactuação de apoio e o arranjo organizacional do apoio matricial e equipe de referência como condições para a promoção da clínica ampliada, que por sua vez embasaria o projeto de saúde no território e o Projeto Terapêutico Singular (PTS). O PTS envolve um conjunto de propostas de condutas terapêuticas articuladas, direcionadas a um indivíduo, família ou coletividade. Tem como objetivo traçar uma estratégia de intervenção para o usuário, contando com os recursos da equipe, do território, da família e do próprio sujeito ${ }^{5}$ e envolve uma pactuação entre esses mesmos atores.

O trabalho em equipe, elemento essencial para a elaboração pactuada e compartilhada do projeto terapêutico, implica em compartilhamento de percepções e reflexões entre profissionais de diferentes áreas do conhecimento na busca pela compreensão da situação ou problema em questão ${ }^{5}$. A construção de um PTS exige a presença e colaboração de sujeitos comprometidos com propostas e condutas terapêuticas articuladas, envolvendo quatro pilares: hipótese diagnóstica, definição de metas, divisão de responsabilidades e reavaliação $0^{6}$.

A elaboração do PTS ocorre em momentos de encontro dos sujeitos, e, na organização dos processos de trabalho do NASF, um desses momentos é o apoio matricial. Apoio matricial é definido como um modelo de organização em que as equipes que atuam junto à população recebem apoio técnico em áreas específicas, sendo um espaço de construção compartilhada para o suporte técnico-pedagógico e assistencial às equipes das unidades de saúde, e o aumento de seu poder resolutivo. $\mathrm{O}$ apoiador matricial deve ser um especialista diferenciado das equipes de SF, em termos de conhecimento e de perfil, contribuindo com saberes e intervenções que aumentem a capacidade resolutiva da equipe de saúde ${ }^{7,8}$.

A maior parte dos estudos sobre o PTS como ferramenta prática no trabalho em saúde mental descreve seu processo de implantação e resultados em serviços com oferta de cuidados mais intensivos ou especializados, como os Centros de Atenção Psicossocial (CAPS) ${ }^{9-11}$. Os resultados dos estudos conduzidos em CAPS mostram o PTS como ferramenta potente no cuidado aos sujeitos atendidos 
por esses serviços, proporcionando o resgate da autonomia e das relações no contexto de vida dos indivíduos e suas famílias. Em pesquisa realizada com profissionais de CAPS, Barros ${ }^{12}$ apontou desafios e dificuldades para a operacionalização do PTS, relacionados à reorganização dos serviços e do trabalho em equipe, à dificuldade de articulação com as redes institucionais e sociais e à inserção dos usuários como participantes efetivos nessa construção.

O emprego do PTS em outros serviços de saúde foi menos estudado e a maior parte do material disponível é relato de experiência. Estudo realizado em um centro de saúde no município de Campinas descreveu o PTS como dispositivo de gestão eficiente ao disparar processos de mudanças nas práticas de saúde e na ampliação das ofertas nos serviços de saúde 5 .

Pela complexidade envolvida e por ser uma das propostas de ampliação do acesso e da integralidade da atenção à saúde, as experiências em saúde mental nos NASF precisam ser acompanhadas e analisadas, já que propostas só são efetivas se transformadas em práticas concretas. Nesse sentido, o objetivo deste estudo é analisar as possibilidades de construção de PTS pelas equipes de saúde mental dos NASF e suas articulações com outros equipamentos da APS, da Atenção Psicossocial e de outros setores da sociedade.

\section{Métodos}

Esta pesquisa foi desenvolvida no município de Guarulhos, estado de São Paulo, cuja população em 2010 era de 1.221.979 habitantes segundo o Censo $^{13}$. É um município com grande contingente de migrantes e marcado por desigualdades socioeconômicas. A rede de saúde guarulhense é dividida em quatro regiões intramunicipais e as regiões apresentam diferenças quanto à distribuição dos serviços de saúde, principalmente dos níveis de atenção secundário e terciário. Em agosto de 2012, a proporção de cobertura pela ESF era de 20,7\% da população total ${ }^{14}$ e a rede de saúde psicossocial era composta por: um CAPS II infantil, um CAPS III Álcool e Drogas, três CAPS II adulto, um CAPS III adulto, um Consultório na Rua, 17 leitos e pronto atendimento em hospital geral e no hospital municipal de urgências.

Após se cadastrar junto ao MS para implantação dos NASF, em outubro de 2009, Guarulhos implantou seis núcleos, distribuídos por três regiões de saúde. Ao limitar o alcance do NASF à ESF, o MS desconsiderou a população abrangida pelas UBS tradicionais (unidades sem saúde da família) e pelas Equipes de Agentes Comunitários de Saúde (EACS). Para dar suporte às UBS tradicionais e EACS, a Secretaria Municipal de Saúde (SMS) de Guarulhos implantou 13 Núcleos de Apoio à Atenção Básica (NAAB), criados na época da implantação e submetidos às mesmas diretrizes dos NASF. Isso possibilitou o incremento de recursos humanos também nessas unidades de saúde, mesmo sem o recebimento de incentivo financeiro federal para esses núcleos.

Foram sujeitos da pesquisa uma equipe NASF e uma NAAB da região de saúde II e uma equipe NASF e uma NAAB da região IV, totalizando quatro núcleos de apoio. A escolha das regiões de saúde foi feita por conveniência, visto que a região I não contava com NASF e optou-se por não envolver a região III por ser território de atuação de uma das pesquisadoras, no intuito de evitar conflitos de interesse. Foram consideradas elegíveis para o estudo equipes com tempo de atuação igual ou superior a seis meses e sem mudanças significativas em sua composição no mesmo período. Em uma das regiões várias equipes preenchiam estes critérios e a escolha foi aleatória; na outra, apenas uma equipe NASF e uma equipe NAAB preencheram os critérios de inclusão.

No município, as categorias componentes da saúde mental nos NASF e NAAB são: psicologia, fonoaudiologia, serviço social e terapia ocupacional. Não há psiquiatras vinculados aos núcleos. Foram realizadas entrevistas individuais e entrevistas semiestruturadas em grupo com os profissionais da saúde mental dos núcleos, visando conhecer o perfil dos profissionais, as atividades que desenvolviam, como se organizavam, como se articulavam com outros recursos de saúde geral e de saúde mental, e as dificuldades encontradas no cotidiano de trabalho. As entrevistas foram gravadas em áudio e transcritas.

Para a apreensão do processo de construção de PTS foi realizada observação sistemática das reuniões de apoio matricial, reuniões de equipe, atividades terapêuticas ou educativas e reuniões com outros setores das quais participaram profissionais da saúde mental dos núcleos de apoio, durante uma semana típica de funcionamento dos núcleos entre agosto e setembro de 2012. A observação foi guiada por um roteiro, que contemplava as atividades realizadas pelos profissionais, suas sequências e conexões, e as tecnologias utilizadas.

Os dados das observações foram organizados em diário de campo e, como as entrevistas, analisados de acordo com três etapas da análise temática ${ }^{15}$ : a) fase de pré-análise, com sistematização dos da- 
dos coletados, através de leituras das transcrições das entrevistas e dos registros de campo; b) fase de exploração do material, em que se identificaram palavras ou expressões relevantes; c) fase de tratamento dos resultados obtidos e interpretação.

Mediante a leitura repetida do material (processo de impregnação) foram identificadas as unidades de registro. Os dados foram organizados em quatro categorias com base nos componentes dos projetos terapêuticos singulares: atuação da equipe (recursos da equipe, transdisciplinaridade e propostas terapêuticas), processo de elaboração do projeto (formulação de hipóteses, definição de metas, responsabilização e reavaliação), estratégias de intervenção com o usuário (recursos do sujeito, da família e do território), e profissional como operador e gestor do cuidado e resolutividade. A análise de conteúdo ${ }^{16}$ foi o método empregado na interpretação.

Os objetivos e procedimentos da pesquisa foram informados às equipes dos serviços e sua realização consentida pelos gerentes. Todos concordaram em fazer parte do estudo e acordaram suas participações mediante assinatura do Termo de Consentimento Livre e Esclarecido (TCLE). O anonimato dos informantes foi garantido. Este estudo foi aprovado pelo Comitê de Ética em Pesquisa da Faculdade de Ciências Médicas Santa Casa de São Paulo.

\section{Resultados e discussão}

\section{Atuação da equipe}

As UBS em que as equipes NAAB e NASF atuavam foram inauguradas em diferentes períodos, da década de 1970 a 2007, e eram bastante distintas em suas estruturas físicas internas e externas. As equipes dos núcleos também eram heterogêneas quanto à população adscrita e sua composição (Tabela 1).

Das 12 UBS observadas, três ofereciam condições adequadas de trabalho às equipes de saúde mental dos núcleos de apoio, no tocante ao espaço físico e materiais necessários ao desenvolvimento de suas atividades. Nas unidades que não dispunham de espaço físico adequado, a equipe dos núcleos de apoio ficava à mercê da ausência de profissionais médicos ou de enfermagem para realizar suas atividades. A carência de recursos físicos era minimizada pela iniciativa dos profissionais de procurarem espaços na comunidade (salas ou salões em igrejas, salão de festas, escola, organização não governamental e biblioteca). Não havia contratualização formal pela SMS e a ocupação dos espaços comunitários não representava parceria ou intervenção no território. Alguns locais eram bastante distantes das UBS e de difícil acesso para os usuários. Outra forma de lidar com a carência de espaços era a centralização das atividades num único local, assim os usuários adscritos em diferentes territórios precisavam se deslocar para uma unidade "sede".

Nenhuma equipe tinha à sua disposição tecnologias de comunicação, tais como telefones ou computadores. A portaria que regulamenta os NASF não prevê destinação de instrumentos ou incentivos de aporte específicos à saúde mental. Recentemente, a Portaria 2.488 de outubro de 2011, que aprova a política nacional de atenção básica, ressalta que é competência do município a "disponibilização de espaço físico adequado nas UBS e a garantia de recursos de custeio necessários ao desenvolvimento das atividades mínimas" ${ }^{17}$ preconizadas em suas diretrizes. Há recomendação expressa para que não seja montada estrutura física específica para as equipes NASF.

Dentre os 16 profissionais de saúde mental dos núcleos pesquisados (cinco psicólogas, duas terapeutas ocupacionais, seis assistentes sociais e três fonoaudiólogas), 13 foram entrevistados individualmente. Foram realizadas quatro entrevistas semiestruturadas em grupo, com participação de, no mínimo, dois profissionais da equipe de saúde mental dos núcleos. Seis profissionais trabalhavam na SMS/Guarulhos antes da implantação dos NASF e NAAB e, desses, apenas três já pertenciam ao quadro da APS. Portanto, a maioria dos profissionais da saúde mental iniciou sua trajetória na APS após o cadastramento do município à Portaria NASF. Nenhum profissional recebeu capacitação para atuar em APS e a maioria afirmou não ter recebido capacitação em saúde mental.

Os núcleos tinham uma composição heterogênea e numericamente diversificada. Havia diferenças quanto à quantidade de unidades $\mathrm{e}$ equipes de saúde da família acompanhadas. Além dos profissionais da área de saúde mental, os demais componentes dos núcleos estudados eram: quatro nutricionistas, quatro educadores físicos e dois fisioterapeutas. Os NAAB eram contemplados com número menor de áreas profissionais. Duas equipes de apoio contavam com cinco profissionais da saúde mental, e outras duas equipes, com três; não foi possível identificar a lógica que estabeleceu essas diferenças.

Todos os núcleos tiveram significativas alterações no quadro geral de profissionais: mais de três mudanças cada um em menos de três anos de 
Tabela 1. População adscrita, unidades básicas de saúde e composição das equipes dos núcleos de apoio, segundo região intramunicipal de saúde de Guarulhos (SP).

\begin{tabular}{|c|c|c|c|c|c|c|c|c|c|c|}
\hline & \multirow[b]{2}{*}{ População $^{\mathrm{a}}$} & \multirow[b]{2}{*}{ UBS } & \multicolumn{8}{|c|}{ Profissionais $^{\mathrm{b}}$} \\
\hline & & & $\begin{array}{l}8 \\
0 \\
0 \\
0 \\
\frac{0}{0} \\
0\end{array}$ & 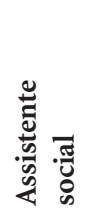 & 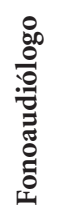 & 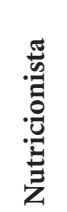 & 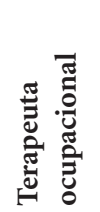 & 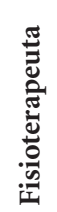 & 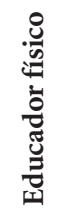 & స్ㅠㅇ \\
\hline \multicolumn{11}{|l|}{ Região I - Centro } \\
\hline \multicolumn{11}{|l|}{ NAAB } \\
\hline Centro & 86.575 & 3 & 4 & 4 & 1 & 0 & 1 & 1 & 1 & 12 \\
\hline Ponte Grande & 47.367 & 4 & 2 & 2 & 0 & 1 & 0 & 1 & 1 & 7 \\
\hline Tranquilidade & 73.775 & 3 & 2 & 3 & 1 & 1 & 1 & 0 & 1 & 9 \\
\hline Cecap & 77.130 & 3 & 3 & 3 & 1 & 1 & 0 & 1 & 1 & 10 \\
\hline Subtotal & 284.847 & 13 & 11 & 12 & 3 & 3 & 2 & 3 & 4 & 38 \\
\hline \multicolumn{11}{|l|}{ Região II - Cantareira } \\
\hline \multicolumn{11}{|l|}{ NAAB } \\
\hline Taboão & 62.899 & 3 & 1 & 1 & 0 & 0 & 0 & 0 & 0 & 2 \\
\hline Jovaia & 110.459 & 3 & 2 & 2 & 1 & 1 & 0 & 0 & 0 & 6 \\
\hline Cabuçu & 29.171 & 4 & 1 & 1 & 0 & 0 & 0 & 1 & 1 & 4 \\
\hline Vila Galvão & 47.125 & 3 & 2 & 1 & 1 & 1 & 0 & 1 & 1 & 7 \\
\hline \multicolumn{11}{|l|}{ NASF } \\
\hline Acácio & 56.438 & 3 & 1 & 1 & 1 & 1 & 2 & 0 & 1 & 7 \\
\hline Continental & 53.111 & 3 & 0 & 0 & 1 & 1 & 0 & 2 & 1 & 5 \\
\hline Subtotal & 359.203 & 19 & 7 & 6 & 4 & 4 & 2 & 4 & 4 & 31 \\
\hline \multicolumn{11}{|c|}{ Região III - São João/Bonsucesso } \\
\hline \multicolumn{11}{|l|}{ NAAB } \\
\hline Inocoop & 50.205 & 3 & 1 & 2 & 1 & 0 & 0 & 0 & 1 & 5 \\
\hline H. Veloso & 53.211 & 2 & 2 & 2 & 1 & 0 & 0 & 0 & 1 & 6 \\
\hline Lavras & 46.786 & 3 & 1 & 2 & 1 & 1 & 0 & 0 & 1 & 6 \\
\hline \multicolumn{11}{|l|}{ NASF } \\
\hline Santos Dumont & 61.077 & 3 & 1 & 1 & 1 & 1 & 0 & 2 & 1 & 7 \\
\hline Soberana & 58.040 & 5 & 2 & 1 & 1 & 1 & 0 & 0 & 1 & 6 \\
\hline Subtotal & 269.319 & 16 & 7 & 8 & 5 & 3 & 0 & 2 & 5 & 31 \\
\hline \multicolumn{11}{|c|}{ Região IV - Pimentas/Cumbica } \\
\hline \multicolumn{11}{|l|}{$\mathrm{NAAB}$} \\
\hline Jandaia & 83.161 & 4 & 1 & 2 & 1 & 0 & 0 & 1 & 1 & 6 \\
\hline Jurema & 73.628 & 4 & 2 & 2 & 1 & 0 & 1 & 0 & 1 & 7 \\
\hline Uirapuru & 40.526 & 3 & 1 & 2 & 0 & 0 & 0 & 0 & 1 & 4 \\
\hline \multicolumn{11}{|l|}{ NASF } \\
\hline Aracília & 49.253 & 5 & 1 & 1 & 1 & 1 & 1 & 0 & 2 & 7 \\
\hline Cummins & 62.042 & 5 & 1 & 1 & 1 & 1 & 0 & 2 & 2 & 8 \\
\hline Subtotal & 308.610 & 21 & 6 & 8 & 4 & 2 & 2 & 3 & 7 & 32 \\
\hline Guarulhos & 1.221 .979 & 69 & 31 & 34 & 16 & 12 & 6 & 12 & 20 & 132 \\
\hline
\end{tabular}

${ }^{\mathrm{a}}$ Fonte: IBGE - CENSO 2010. ${ }^{\mathrm{b}}$ Fonte: Secretaria Municipal de Saúde - Guarulhos (SP). Informações referentes a 2012.

implantação. Os dois NASF pesquisados recebiam supervisão técnica desde o primeiro semestre de 2012, ao passo que os NAAB não contavam com esse apoio até o final das observações.

\section{Processo de elaboração do PTS}

Foram observadas 15 reuniões de apoio matricial, das quais 10 envolveram as equipes das UBS e as equipes NASF ou NAAB, três contaram também com a participação de um psiquiatra diretamente ligado à Supervisão de Saúde e duas envolveram 
alguns profissionais de CAPS. Em geral as equipes dos núcleos se apresentaram completas ou quase completas nas reuniões. Os gerentes e os médicos de UBS apenas participaram de reuniões com presença do psiquiatra ou da equipe do CAPS. O número de Agentes Comunitários de Saúde (ACS) presentes às reuniões variou de um ou dois profissionais a toda equipe.

Foram relatados 187 casos durante as reuniões, relatados por enfermeiros ou auxiliares de enfermagem, que estiveram presentes nas reuniões quando tinham sido responsáveis pelo acolhimento dos usuários, ou pelo profissional NASF. Poucos casos foram relatados diretamente pelos ACS ou pelos médicos. Foi significativo o número de casos encaminhados por médicos da própria equipe da UBS.

A maior parte dos casos eram crianças, seguidos de mulheres, adolescentes e, por último, de homens. Foram explicitadas condutas para 119 casos e, em 111 deles, o manejo seria conduzido pela equipe de saúde mental dos núcleos. As condutas para as crianças foram, em geral, encaminhamento para grupos de avaliação, de orientação a pais ou de família. Para os adultos, foram indicados atendimentos individuais, inclusão em grupos terapêuticos ou realização de visita domiciliar.

Os grupos indicados eram coordenados e conduzidos pelos profissionais de saúde mental dos núcleos. Houve indicação para apenas um grupo conduzido pela equipe de saúde da própria UBS e uma indicação de conduta compartilhada, restrita a atendimento por dois profissionais do próprio núcleo.

A ausência de diferentes profissionais nas reuniões, notoriamente dos médicos das UBS, pode colaborar para dificultar o vínculo entre as equipes e inviabilizar a troca de informações e discussão de casos. A organização de fluxo de atendimento ao usuário em etapas: 1. Encaminhamento por escrito ao especialista do NASF ou NAAB; 2 . Acolhimento por um profissional da equipe da UBS; 3. Discussão em reunião de apoio matricial; 4. Estabelecimento de conduta e, 5 . Atuação da equipe de apoio; assemelha-se a uma prática burocrática que entrava as relações de trabalho e que fortalece a lógica do encaminhamento para o especialista, agora mais acessível.

Observaram-se momentos em que a reunião se limitou à passagem de guias de encaminhamento, sem questionamento ou informação sobre o usuário além da queixa manifesta e uma reunião de apoio matricial sem nenhum membro da equipe de saúde da UBS. Assim, as reuniões de apoio matricial, o locus principal de elaboração de PTS por sua característica de momento de encontro das equipes, mostrou-se um espaço silencioso de qualquer discussão técnico-pedagógica interdisciplinar, apesar da composição multiprofissional das equipes dos NASF e NAAB e da possibilidade de participação de diferentes profissionais das UBS.

A presença de diversos profissionais de diferentes áreas foi insuficiente para qualificar as discussões; em alguns momentos, foi visível clima de constrangimento, desconfiança e distanciamento entre as equipes. A reprodução de práticas de encaminhamento e dos modelos de conhecimento técnico sobre os transtornos mentais tem sido apontada como obstáculo à construção do cuidado em saúde mental na APS ${ }^{18,19}$. Essa reprodução é uma redução das possibilidades do apoio matricial (gerencial-administrativa; político-institucional; técnico-pedagógico; técnico-assistencial e político-comunitário) como ferramenta para a promoção do cuidado ${ }^{20}$.

As observações realizadas, complementadas por dados das entrevistas, evidenciaram uma relação pautada por pouca articulação e comunicação entre os profissionais dos núcleos de apoio, os profissionais da saúde das UBS e os demais equipamentos de saúde e de saúde mental. Essa situação foi observada em UBS de SF e em UBS tradicionais e pode ter raízes na forma de implantação dos NASF e NAAB, na falta de capacitação para o trabalho e de clareza quanto às atribuições das equipes de apoio, como relatado na fala abaixo:

Foi uma semana de treinamento, mas sem a minima apresentação, na verdade a gente foi fazendo por si, o grupo foi fazendo, estudando, foi o tempo que a gente teve para pegar material, para ler, a gente não teve nenhum curso de formação. (Profissional 6).

A presença não sistemática da equipe de apoio nas unidades de saúde, em consequência da grande quantidade de equipamentos e/ou equipes de saúde da família sob sua responsabilidade ou pelo excesso de reuniões com outras instâncias, surgiu como um obstáculo à vinculação das equipes. Os profissionais da UBS convivem diariamente, enquanto a equipe de apoio comparece eventualmente às unidades ${ }^{12,21}$ (por exemplo, embora a equipe de um NASF frequentasse uma UBS uma vez por semana, interagia somente uma vez ao mês com cada equipe da unidade). Além disso, a equipe dos núcleos está sobrecarregada de atividades e essa situação dificulta que as equipes se percebam como uma só equipe em integração $0^{22}$ que possam colaborar mutuamente, fazer solicitações concretas ao outro e compartilhar dúvidas e conhecimentos. 


\section{Estratégias de intervenção com o usuário}

Nas entrevistas, os profissionais da saúde mental identificaram que as queixas de muitos usuários estavam relacionadas aos vínculos familiares e às condições de vida dos sujeitos, o que exigiria uma intervenção em rede de saúde e intersetorial. Nas reuniões de apoio matricial, essa associação esteve ausente ou apareceu muito enfraquecida, sem possibilitar a elaboração de estratégias de intervenção que considerassem a família e o território. Algumas situações foram direcionadas a condutas pré-definidas: independentemente de discussão, de acordo com a queixa, formulava-se a conduta, e o usuário era direcionado para algum grupo coordenado e conduzido por um ou mais profissionais da equipe de saúde mental dos NASF ou NAAB, ou para atendimento individual restrito a um dos profissionais dos núcleos. Em nenhum momento foi explicitada a rediscussão das condutas com o sujeito e/ou a família, resultado semelhante ao relatado em estudo conduzido no município de Sobral, Cearáa ${ }^{23}$.

Diversas queixas relatadas traziam explícitas questões relacionadas às precariedades econômicas e sociais geradoras de violência. $\mathrm{O}$ enfrentamento a situações de violência e de ruptura social não envolveu articulação intersetorial (ao contrário do preconizado pelas diretrizes), seja pela percepção de falta de respaldo organizacional e sensação de impotência, seja pela percepção de que isso não cabe à saúde, portanto, não há o que ser feito: a situação foge da competência da UBS... (profissional 1 da UBS); se a família não traz, não há o que fazer (profissional 2 da UBS); não temos respaldo (profissional da educação em reunião intersetorial).

A experiência de elaboração e execução de PTS entre uma equipe de saúde mental com a equipe de uma escola pode ilustrar as potencialidades e desafios encontrados pelos profissionais nessa trajetória, tanto no que se refere à composição de equipe quanto em termos de prática voltada para o cuidado em saúde mental: em longa reunião, discutiu-se o caso de uma criança que apresentava comportamento agressivo e antissocial e se encontrava em atendimento individual com a psicóloga do núcleo. As equipes analisaram os recursos da escola, da família e da criança e elaboraram um plano estratégico envolvendo a família, entidades sociais e jurídicas.

Em outra reunião realizada apenas com representantes de equipes de saúde mental NASF e NAAB, de uma região de saúde, e a equipe de referência de um CAPS infanto-juvenil houve discussão e compartilhamento de aspectos sociais, psicológicos e clínicos que envolviam os casos.

De maneira geral, porém, não se observou preparação dos casos levados à reunião de apoio matricial. As descrições muitas vezes se restringiram à leitura da queixa manifestada pelo usuário, as hipóteses se limitaram ao diagnóstico psiquiátrico e não houve estabelecimento de metas. A reavaliação dos casos restringiu-se à mudança na conduta terapêutica medicamentosa ou à proposta de rediscussão em novo momento de apoio matricial.

Durante as reuniões, a percepção foi de que o caso relatado ficaria sob responsabilidade do profissional que levou o caso para discussão ou o que atenderia, sem uma análise das condições desse profissional para colaborar na articulação das informações, na articulação com os serviços e na atuação como referência para o usuário e/ou família e/ou comunidade.

Esses achados são semelhantes aos de um estudo sobre os NASF realizado em Campina Grande (PB) em que se observou que as atuações dos profissionais do NASF eram majoritariamente isoladas, de acordo com o seu núcleo de saber disciplinar ${ }^{24}$. Segundo os autores, as diretrizes ministeriais não possibilitaram a consolidação do novo modelo de saúde proposto para o apoio à APS, pois deram espaço para que modos de atenção tradicionais e fragmentados se mantivessem operantes no interior dos NASF.

Os relatos dos acolhimentos aos usuários feitos pelos profissionais das UBS caracterizaram-se pela escassez de informações a respeito da dinâmica de vida do sujeito e situações em que era perceptível o sofrimento ou identificação direta do acolhedor com a história ou queixa relatada, caracterizando despreparo e possibilidade de adoecimento do profissional que realizou o acolhimento.

\section{O profissional como operador e gestor do cuidado e resolutividade}

As equipes de saúde mental dos núcleos referiram diversas iniciativas de implantação de trabalhos com grupos, sendo que alguns finalizaram por falta de adesão. O descontentamento e a sensação de desvalorização profissional, associados a condições de trabalho adversas e de muitas exigências podem afastar o profissional do objeto de seu trabalho. Essa situação é evidenciada na entrevista abaixo:

... sinto o meu trabalho sendo em vão, muito sem objetivo, como se eu estivesse ali tapando buraco, porque não tem outra coisa para eles. Eu vou vendo 
eles melhorando, mas, às vezes, fico pensando, ah, tá, é isso aqui mesmo que tá resolvendo isso?, tô fazendo alguma coisa?... não tem propósito. (Profissional 9)

$\mathrm{O}$ apoio grupal e a confiança na equipe foram apontados como aspectos positivos para a continuidade e a constante renovação de credibilidade no trabalho, indicando potencialidade para constituição de trabalho de equipe em integraçãa ${ }^{22}$, desde que não se restrinja somente à equipe do núcleo de apoio. Em contraposição, foram manifestadas situações de isolamento dos profissionais, tanto em relação às equipes de saúde quanto à própria equipe NASF e NAAB:

... eu me sinto um pouco sozinha, acho que essa dificuldade, de não ter muito com quem dividir o trabalho ou então de não ter com quem discutir caso..., às vezes, você não sabe o que fazer em determinada situação... tem algumas coisas que a gente compartilha, a maior parte não, cada um fica no seu quadrado. (Profissional 4)

As diretrizes imputam aos profissionais NASF além da cogestão do cuidado, a execução de ações de cura, reabilitação, prevenção e promoção da saúde. Diante da diversidade e quantidade de demandas, os profissionais de saúde mental NASF e $\mathrm{NAAB}$ enfrentam grande dificuldade em organizar seu trabalho.

A equipe de apoio é em si mesma uma junção de profissionais de áreas, formações e trajetórias diversas. O manejo dessas diferenças defronta-se com o desafio de compor com as demais equipes (UBS, rede de saúde e psicossocial) e com a exigência de que executem atividades terapêuticas, educativas e de formação e capacitação. Além de fomentarem as discussões de casos com o objetivo de operacionalizar PTS, são convocados a colaborarem ou desenvolverem ações voltadas para públicos específicos, tais como de planejamento familiar, grupos de hipertensos e diabéticos, gestantes, saúde do escolar, usuários acamados etc., conforme já investigado por diversos estudos ${ }^{1,5}$.

Não foram observadas diferenças significativas entre NASF e NAAB na construção do PTS junto às equipes de saúde das UBS. Contudo, foram observadas dificuldades de diversas ordens, assemelhando-se às descrições dos obstáculos constatados por Campos e Domitti ${ }^{25}$ : estruturais (referentes à maneira como as organizações se conformam, favorecendo ou não o cuidado interdisciplinar e dialógico); epistemológicos (relativos à racionalidade predominante das especialidades, que gera o pensar e o agir numa perspectiva restrita) e éticos (relacionados ao sigilo).

Além disso, há o excesso de demanda associado à carência de recursos, às dificuldades de comu- nicação em todas as instâncias da rede de saúde e rede psicossocial e o imperativo de consciência profissional quanto à grande demanda por atendimentos individuais em contraposição às diretrizes que orientam que os atendimentos sejam, em sua maioria, grupais. Apesar das demonstrações de desejo de mudança, os profissionais são interrogados e se interrogam sobre como operar as diretrizes ministeriais ao resvalarem em diversos tipos de obstáculos e precisam lidar diariamente com a distância entre o prescrito e a realidade ${ }^{26}$.

Nas observações e nas entrevistas realizadas foi possível identificar dificuldades de articulação em rede psicossocial, de saúde em geral e com as instâncias sociais legitimadas pelo próprio SUS como componentes da rede, tais como conselhos gestores das UBS e escolas. Do mesmo modo, também os recursos comunitários não foram mencionados nas discussões dos casos e não foram articulados às condutas definidas. Essa desarticulação pode estar associada à inexistência de recursos no território, ao desconhecimento dos recursos existentes pelas equipes ou a não consolidação do pensar e atuar em rede.

Apesar dos avanços proporcionados pela implantação do NASF em Guarulhos, em especial pelo grande incremento de recursos humanos, com inclusão de novas profissões que não faziam parte do quadro funcional da APS, e pelo aumento numérico de profissionais que eram escassos, é necessária uma revisão crítica deste processo, visando alcançar novos patamares de cuidado e novas práticas de saúde mental.

\section{Triangulação APS/NASF/CAPS}

É oportuno reavaliar a quem cabe o quê na formulação e construção de PTS na triangulação APS/NASF/CAPS. A APS é a porta de entrada no sistema nacional de saúde para quaisquer pessoas e os CAPS são a porta de entrada para as pessoas com transtornos mentais graves de longa evolução; a ambos é atribuída a função de coordenação do cuidado. Por outro lado, de acordo com a Portaria 154, o NASF também exerceria a função de coordenação, além de atuar na assistência direta. Existe uma sobreposição de funções entre NASF e $\mathrm{CAPS}^{27}$ : o CAPS é originalmente um equipamento de atenção, mas entre suas funções está a execução do apoio matricial à APS, e o NASF, que prioritariamente deveria fazer o apoio matricial, atua fortemente na atenção. Essa sobreposição de funções gera indefinição de papéis, fomenta divergências e expõe contradições de origem. Ora ambos competem, ora fica um vazio. 
O papel do PTS como ferramenta de cuidado em saúde em CAPS já foi analisado ${ }^{12,28} \mathrm{e}$ estudos de implantação do PTS na APS $^{5}$ sugerem sua viabilidade tecnológica. No entanto, é imperativo analisar sua viabilidade a partir das peculiaridades da $\mathrm{APS}^{8}$, evitando a mera transposição de ferramentas tecnológicas entre serviços componentes de uma rede de atenção psicossocial, distintos em diversos aspectos.

Entre essas distinções podemos destacar:

- No CAPS, o PTS pressupõe uma relação entre uma equipe de cuidadores e um usuário que pode ser um sujeito, uma família ou um grupo maior. O dispositivo NASF introduz uma mudança nesse cenário, porque há uma equipe de saúde, um usuário, uma segunda equipe (a equipe NASF). Estabelece-se uma relação "em degrau", em que a equipe NASF apoia a equipe da UBS para que esta elabore e desenvolva ações de cuidado no campo da saúde mental. E ao CAPS cabe também a elaboração e desenvolvimento de ações com seus usuários no território, implicando o envolvimento das equipes de saúde das UBS e NASF na sua execução.

- Existe grande diferença entre a clientela atendida pela APS e a atendida pelo CAPS: o CAPS cuida prioritariamente de pessoas com transtornos mentais graves de longa evolução, um público delimitado e que representa uma proporção pequena da população. A APS cuida de uma clientela maior e extremamente heterogênea quanto às demandas, à faixa etária, ao diagnóstico.

- As pessoas com transtornos mentais que não se caracterizem como graves deveriam ser tratadas no âmbito da APS, seja pela ESF ou em UBS tradicional. A APS conta com poucos profissionais de saúde mental que possam atender e cuidar de pessoas com os denominados transtornos mentais comuns $^{29}$, cujo manejo pode ser complexo e levar à incapacidade funcional ${ }^{30}$. Em princípio, esses recursos humanos mais preparados estão lotados nos NASF, que passam a assumir o apoio matricial, juntamente com o cuidado de um grande número de usuários. Nessas circunstâncias, desviado de sua função principal, a equipe do NASF ocupa boa parte do tempo em atividades de atenção.

- O PTS de um sujeito atendido em CAPS é um projeto de longa duração, porque essas pessoas apresentam problemas e necessidades que em geral demandam longo tempo de cuidado para obtenção de resultados ou, pelo menos, não se têm expectativa de resolutividade a curto prazo. O público da APS, em sua maioria, tem questões que demandam intervenções imediatas e de rápida resoluçãa ${ }^{27,29}$. Isso exige das equipes, tanto das UBS quanto de saúde mental NASF, uma grande agilidade para reavaliar e eventualmente propor novos projetos. Além disso, como a clientela da UBS é composta por pessoas que estão ativamente na vida (trabalhando, estudando, consumindo, se relacionando), a interação com outros equipamentos de saúde e com recursos comunitários e sociais precisa ser mais intensa e ágil, seja com a justiça, a educação, a cultura, o esporte, com a segurança pública ou com a assistência social.

Por fim, os profissionais que atuam no NASF não receberam capacitação ou formação para atuar na instância de apoio à gestão, são profissionais que foram formados para exercer o cuidado e, por isso, têm maior facilidade para executar ações de atenção do que de gestão. Além disso, a qualificação em saúde mental aos profissionais de áreas não associadas tradicionalmente à saúde mental é necessária para aumentar sua capacidade de tomar decisões e agir.

\section{Considerações finais}

A indefinição de objeto de trabalho (atenção, apoio matricial ou apoio à gestão?) e a precariedade das condições de trabalho dificultam a implantação de ações que tenham como foco a promoção à saúde e a prevenção de doenças, deixando à deriva as diretrizes da APS e do NASF, como a integralidade, a interdisciplinaridade, a territorialidade, a articulação em rede de saúde e psicossocial e o incentivo à participação social.

A interpretação dos achados desta pesquisa deve levar em conta o fato de que a observação sistemática foi restrita a uma semana de acompanhamento de cada equipe dos núcleos. A proximidade da pesquisadora que realizou as observações e entrevistas (AAH), também atuante na rede de serviços do município, pode ter acarretado irreais expectativas dos profissionais sobre os possíveis efeitos da pesquisa no seu trabalho e induzido mudanças nas informações dadas durante as entrevistas e no seu comportamento durante as atividades. Todavia, essa proximidade pode estar relacionada também à boa receptividade das equipes dos núcleos e das UBS na realização da pesquisa.

A operacionalização do PTS, principalmente em contextos de equipes em que essa ferramenta tecnológica não está incorporada, pode gerar tensões entre seus componentes e divergências quanto a sua importância, e até mesmo, consciente ou inconscientemente, a criação de obstáculos para obtenção de informações importantes para a compreensão do problema. Outro entrave é a 
situação de equipes de saúde nas quais o processo de trabalho do equipamento inviabiliza ou dificulta os momentos de encontros e a ocorrência de processos em que os profissionais são mais valorizados pela quantidade de procedimentos e atendimentos que pela qualidade e resolutividade das intervenções ${ }^{1}$.

Novos arranjos e esforços devem ser feitos, com a finalidade de reformular o processo de trabalho das equipes de saúde das UBS, dos núcleos de apoio e aumentar sua capacidade de dar resposta às necessidades de saúde mental da população. É necessário analisar os efeitos das transformações propostas pelas diretrizes NASF sobre a realidade da APS: os dilemas e desafios dos profissionais dos núcleos, entre o que deve e o que pode ser feito em relação ao cuidado às pessoas com transtornos mentais, e a compreensão do NASF como uma estratégia de gestão e de reorganização do processo de trabalho na APS.

\section{Colaboradores}

AA Hori trabalhou na elaboração do projeto, coleta e análise dos dados e redação do manuscrito; AF Nascimento, na elaboração do projeto, análise dos dados e redação do manuscrito. 


\section{Referências}

1. Dimenstein M, Santos YF, Brito M, Severo AK, Morais C. Demanda em saúde mental em unidades de saúde da família. Mental 2005; 3(5):23-41.

2. Tanaka OY, Ribeiro EL. Mental health in primary care: ways to reach an integral care. Cien Saude Colet 2009; 14(2):477-486.

3. World Health Organization (WHO). Relatório sobre a saúde no mundo: Saúde mental: nova concepção, nova esperança. Lisboa: WHO; 2002.

4. Brasil. Portaria GM 154 de 24 de janeiro de 2008. Cria os Núcleos de Apoio à Saúde da Família - NASF. Diário Oficial da União 2008; 24 jan.

5. Oliveira GN. O Projeto terapêutico como contribuição para a mudança das práticas de saúde [dissertação]. Campinas: Universidade Estadual de Campinas; 2007.

6. Brasil. Ministério da Saúde (MS). Política Nacional de Humanização. Clínica Ampliada, equipe de referência e projeto terapêutico singular. 2a ed. Brasília: MS; 2008.

7. Campos GWS. Equipes de referência e apoio especializado matricial: um ensaio sobre a reorganização do trabalho em saúde. Cien Saude Colet 1999; 4(2):393-403.

8. Cunha GT. A construção da clínica ampliada na atenção básica. São Paulo: Hucitec; 2005.

9. Carvalho LGP, Moreira MDS, Rézio LA, Teixeira NZF. A construção de um Projeto Terapêutico Singular com usuário e família: potencialidades e limitações. O Mundo da Saúde 2012; 36(3):521-525.

10. Pinto DM, Jorge MSB, Pinto AGA, Vasconcelos MGF, Cavalcante CM, Flores AZT, Andrade AS. Projeto Terapêutico Singular na Produção do Cuidado Integral: Uma Construção Coletiva. Texto \& Contexto Enfermagem 2011; 20(3):493-502.

11. Mororó ME, Colvero LA, Machado AL. The challenges of comprehensive care in a Psychosocial Care Center and the development of therapeutic projects. Rev Esc Enferm USP 2011; 45(5):1171-1176.

12. Barros JO. A construção de projetos terapêuticos no campo da saúde mental: apontamentos acerca das novas tecnologias de cuidado [dissertação]. São Paulo (SP): Universidade de São Paulo; 2010.

13. Instituto Brasileiro de Geografia e Estatística (IBGE). Censo 2010. [acessado 2012 jun 5]. Disponível em: http://www.ibge.gov.br/cidadesat/painel/painel.php?codmun $=35188$

14. Brasil. Ministério da Saúde (MS). Departamento de Atenção Básica. Teto, credenciamento e implantação das estratégias de Agentes Comunitários de Saúde, Saúde da Família e Saúde Bucal. Brasília: MS; 2012 [acessado 2013 apr 04]. Disponível em: http://dab.saude.gov.br/ dab/historico_cobertura_sf/historico_cobertura_sf_ relatorio.php.

15. Minayo MCS. O Desafio do Conhecimento. $2^{\text {a }}$ ed. São Paulo: Hucitec-Abrasco; 1993.

16. Bardin L. Análise de conteúdo. $4^{\mathrm{a}}$ ed. Lisboa: Edições 70; 2006.

17. Brasil. Ministério da Saúde (MS). Portaria 2.488 de 21 de outubro de 2011. Aprova a Política Nacional de Atenção Básica e estabelece a revisão de diretrizes e normas para a organização da Atenção Básica, para a Estratégia Saúde da Família e para o Programa de Agentes Comunitários de Saúde. Diário Oficial da União 2011; 22 out.
18. Lima AIOL, Severo AK, Andrade NdL, Soares GP, Silva LM. O desafio da construção do cuidado integral em saúde mental no âmbito da atenção primária. Temas em Psicologia 2013; 21(1):71-82.

19. Freire FMS, Pichelli AAWS. O psicólogo apoiador matricial: percepções e práticas na atenção básica. Psicologia: Ciência e Profissão 2013; 33(1):162-173.

20. Sampaio J, Martiniano CS, Rocha AMO, Souza Neto AA, Oliveira Sobrinho GD, Marcolino EC, Magalhães FC, Souza FF. Núcleo de Apoio à Saúde da Família: refletindo sobre as acepções emergentes da prática. Revista Brasileira de Ciências da Saúde 2013; 17(1):47-54

21. Vannucchi AMC. Modalidades tecnoassistenciais e atuação do médico psiquiatra no campo da atenção primária à saúde no contexto atual do Sistema Único de Saúde (SUS) [dissertação].São Paulo: Faculdade de Ciências Médicas da Santa Casa de São Paulo; 2011.

22. Peduzzi M. Trabalho em equipe de saúde no horizonte normativo da integralidade, do cuidado e da democratização das relações de trabalho. In: Pinheiro R, Barros MEB, Mattos RA, organizadores. Trabalho em Equipe sob o Eixo da Integralidade: valores, saberes e práticas. Rio de Janeiro: IMS/UERJ, CEPESC, Abrasco; 2007. p. 161-177.

23. Sousa MLT, Tófoli LFF. Apoio matricial em saúde mental na atenção primária: acesso e cuidado integral. Cadernos ESP 2012; 6(2):13-21.

24. Sampaio J, Sousa CSM, Marcolino EC, Magalhães FC, Souza FF, Rocha AMO, Souza Neto AA, Sobrinho GDO. O NASF como dispositivo da gestão: limites e possibilidades. Revista Brasileira de Ciências da Saúde 2012; 16(3):317-324.

25. Campos GW, Domitti AC. Matrix support and reference team: a methodology for interdisciplinary health work management. Cad Saude Publica 2007; 23(2):399-407.

26. Dejours C. Subjetividade, trabalho e ação. Revista Produção 2004; 14(3):27-34.

27. Dimenstein M, Macedo JP. Formação em Psicologia: requisitos para atuação na atenção primária e psicossocial. Psicologia: Ciência e Profissão 2012; 32(n. especial):232-245.

28. Boccardo ACS, Zane FC, Rodrigues S, Mângia EF. O projeto terapêutico singular como estratégia de organização do cuidado nos serviçosde saúde mental. Revista de Terapia Ocupacional da Universidade de São Paulo 2011;22(1):85-92.

29. Onocko-Campos R, Gama CAP. Saúde mental na atenção básica. In: Campos GWS, Guerrero AVP, organizadores. Manual de práticas em atenção básica: saúde ampliada e compartilhada. São Paulo: Hucitec; 2008.

30. Maragno L, Goldbaum M, Gianini RJ, Novaes HM, César CL. Prevalence of common mental disorders in a population covered by the Family Health Program (QUALIS) in São Paulo, Brazil. Cad Saude Publica 2006; 22(8):1639-1648.

Artigo apresentado em 11/07/2013

Aprovado em 01/10/2013

Versão final apresentada em 11/10/2013 